\title{
Adiabatic index of dense matter and damping of neutron star pulsations
}

\author{
P. Haensel ${ }^{1}$, K. P. Levenfish ${ }^{2}$, and D. G. Yakovlev ${ }^{2}$ \\ 1 N. Copernicus Astronomical Center, Bartycka 18, 00-716 Warszawa, Poland \\ e-mail: haensel@camk.edu.pl \\ 2 Ioffe Physical Technical Institute, Politekhnicheskaya 26, 194021 St.-Petersburg, Russia \\ e-mail: ksen@astro.ioffe.rssi.ru;yak@astro.ioffe.rssi.ru
}

Received 17 June 2002 / Accepted 30 July 2002

\begin{abstract}
The adiabatic index $\Gamma_{1}$ for perturbations of dense matter is studied under various physical conditions which can prevail in neutron star cores. The dependence of $\Gamma_{1}$ on the composition of matter (in particular, on the presence of hyperons), on the stellar pulsation amplitude, and on the baryon superfluidity is analyzed. Timescales of damping of stellar pulsations are estimated at different compositions, temperatures, and pulsation amplitudes. Damping of pulsations by bulk viscosity in the neutron-star cores can prevent the stars to pulsate with relative amplitudes $\gtrsim(1-15) \%$ (depending on the composition of matter).
\end{abstract}

Key words. stars: neutron - dense matter - oscillations

\section{Introduction}

The adiabatic index $\Gamma_{1}=\left(n_{\mathrm{b}} / P\right)\left(\mathrm{d} P / \mathrm{d} n_{\mathrm{b}}\right)$ for density perturbations determines the changes of pressure $P$ associated with variations of the local baryon density $n_{\mathrm{b}}$ (e.g., Shapiro $\&$ Teukolsky 1983). It enters the equations governing smallamplitude neutron-star pulsations (Thorne \& Campolattaro 1967; Thorne 1968, 1969a,b) as well as the criteria of stability of cold relativistic stars (Meltzer \& Thorne 1966; Chanmugan \& Gabriel 1971; Chanmugan 1977; Gourgoulhon et al. 1995). For the time-dependent perturbations, like neutron-star pulsations, $\Gamma_{1}$ has to be calculated taking into account the slowness of various equilibration channels in dense matter. The same factors strongly affect viscous damping of neutron-star pulsations.

In the present paper, we study three main factors which regulate $\Gamma_{1}$ and the viscous damping of pulsations: composition of dense matter, pulsation amplitude, and superfluidity of baryons. Equilibration processes for various compositions of neutronstar cores are studied in Sect. 2. In Sect. 3, we calculate the adiabatic index under various conditions of density, composition, and temperature. We separately study two different regimes: first, the regime with the perturbations of chemical potentials of particles much smaller than $T$, and second, the regime with the perturbations much larger than $T$ (we use the units in which the Boltzmann constant $k_{\mathrm{B}}=1$ ); we also consider the effect of baryon superfluidity. In Sect. 4 we estimate the timescales of the viscous damping of density pulsations under various physical conditions which can be realized in the neutron-star

Send offprint requests to: P. Haensel, e-mail: haensel@camk . edu.pl cores. Finally, in Sect. 5 we summarize our results and briefly discuss the problems which remain to be solved.

\section{Relaxation processes in neutron-star cores}

It is widely believed that dense matter of the outer neutron-star core $\left(0.5 n_{0} \lesssim n_{\mathrm{b}} \lesssim 2 n_{0}\right.$, where $n_{0} \approx 0.16 \mathrm{fm}^{-3}$ is the number density of saturated nuclear matter) contains neutrons (n), protons (p), electrons (e), and - if the electron Fermi energy exceeds the muon rest energy (that occurs at $n_{\mathrm{b}} \gtrsim n_{0}$ ) - also muons $(\mu)$. At higher densities in the inner core, hyperons may appear, first of all, $\Sigma^{-}$and $\Lambda$ hyperons. All constituents of matter are strongly degenerate. We restrict ourselves to this composition and will not consider exotic models of matter containing pion or kaon condensates or free quarks. We will study a neutrino-transparent neutron-star core formed in about $30 \mathrm{~s}$ after the neutron star birth in a supernova explosion. We ignore thus the early stage of the neutrino opaque core in a protoneutron star (the adiabatic index in such matter was studied by Gondek et al. 1997).

\subsection{Strong and weak-interaction processes}

Full thermodynamic equilibrium in dense matter is established only after partial equilibria are achieved in all equilibration channels. In our case, there may be five types of channels (labelled as (a), (a'), (b), (c), and (d)), with drastically different relaxation times. These channels, described partly, e.g., 
by Yakovlev et al. (2001) and Haensel et al. (2002), are as follows:

(a) Strong and Coulombic elastic collisions,

$\mathrm{BB}^{\prime} \rightleftharpoons \mathrm{BB}^{\prime}, \quad \ell \mathrm{B} \rightleftharpoons \ell \mathrm{B}, \quad \ell \ell^{\prime} \rightleftharpoons \ell \ell^{\prime}$,

where $\mathrm{B}$ and $\mathrm{B}^{\prime}$ are baryons (nucleons $\mathrm{B}=\mathrm{N}$ or hyperons $\mathrm{B}=\mathrm{H}$ ), while $\ell$ and $\ell^{\prime}$ are leptons in dense matter (electrons or muons). These processes do not change particle fractions.

$\left(\mathrm{a}^{\prime}\right)$ Strong-interaction processes conserving strangeness but changing baryon fractions,

$\mathrm{n} \Lambda \rightleftharpoons \mathrm{p} \Sigma^{-}$.

(b) Modified Urca processes like

$\mathrm{nN} \rightarrow \mathrm{N}^{\prime} \mathrm{p} \ell \bar{v}_{l}, \quad \mathrm{pN} \ell \rightarrow \mathrm{nN}^{\prime} v_{\ell}$

where $v_{\ell}$ and $\bar{v}_{\ell}$ are neutrino and antineutrino. Similar processes can also proceed with one or both nucleons replaced by hyperons. These processes can change the fractions of nucleons, hyperons, and leptons.

(c) Direct Urca processes involving nucleons and hyperons,

$\mathrm{n} \rightarrow \mathrm{p} \ell \bar{v}_{\ell}, \quad \mathrm{p} \ell \rightarrow \mathrm{n} v_{\ell}$

$\Sigma^{-} \rightarrow \mathrm{n} \ell \bar{v}_{\ell}, \quad \mathrm{n} \ell \rightarrow \Sigma^{-} v_{\ell}$

$\Lambda \rightarrow \mathrm{p} \ell \bar{v}_{\ell}, \quad \mathrm{p} \ell \rightarrow \Lambda v_{\ell}$

which, similarly to processes (b), can change the fractions of nucleons, hyperons, and leptons.

(d) Nonleptonic collisions of baryons changing strangeness, e.g.,

$\mathrm{N} \Lambda \rightleftharpoons \mathrm{Nn}, \quad \mathrm{nn} \rightleftharpoons \mathrm{p} \Sigma^{-}$

they can change the fractions of baryons, but do not affect the fractions of leptons.

\subsection{Relaxation times}

Relaxation times in channels (a) and $\left(\mathrm{a}^{\prime}\right)$ are about $\left(10^{-16}-10^{-19}\right) / T_{9}^{2}$ s or shorter, where $T_{9} \equiv T / 10^{9} \mathrm{~K}$ (this can be deduced, e.g., from the results of Flowers \& Itoh 1979). Therefore, these channels guarantee instantaneous (on hydrodynamical time scales) partial thermodynamic equilibration to the Fermi-Dirac distributions of particles of all species $j$ with a temperature $T$ and chemical potentials $\mu_{j}$. Processes ( $\left.\mathrm{a}^{\prime}\right)$ allow for additional equilibration of $\Lambda, \Sigma^{-}$, and nucleons, which leads to the equality of the chemical potentials $\mu_{\Sigma}+\mu_{\mathrm{p}}=\mu_{\mathrm{n}}+\mu_{\Lambda}$. The relaxation in channels (b), (c), and (d) (with relaxation times $\tau_{\mathrm{M}}, \tau_{\mathrm{D}}$, and $\tau_{\mathrm{H}}$ ) is associated with weak interactions and lasts, therefore, much longer; it will be important for our analysis. In a nonsuperfluid matter close to full equilibrium the relaxation times can be estimated as (e.g., Yakovlev et al. 2001; Haensel et al. 2002)

$\tau_{\mathrm{M}} \sim \frac{2 \text { month }}{T_{9}^{6}}, \quad \tau_{\mathrm{D}} \sim \frac{20 \mathrm{~s}}{T_{9}^{4}}, \quad \tau_{\mathrm{H}} \sim \frac{1 \mathrm{~ms}}{T_{9}^{2}}$.
These estimates are valid if only the pulsation amplitudes $\delta \mu_{j} \equiv$ $\left|\mu_{j}-\mu_{j}^{(0)}\right|$ are very low, $\delta \mu_{j} \ll T$, where $\mu_{j}^{(0)}$ is the fully equilibrium value of $\mu_{j}$. We will call this regime as the regime of subthermal pulsations.

According to Eq. (8), the slowest are modified Urca processes which involve neutrinos and largest number of strongly degenerate particles. The most rapid are nonleptonic processes changing strangeness; they go via weak interaction but involve smallest number of degenerate particles and no neutrinos. While estimating $\tau_{\mathrm{H}}$ and viscous dissipation parameters in hyperonic matter from the results of Haensel et al. (2002), we set the phenomenological hyperon reaction parameter $\chi=0.1$. All the parameters in hyperonic matter are rather uncertain and can differ from the presented values within a factor of ten or even larger.

\section{Adiabatic index}

Clearly, one can divide the neutron star core into three zones with different relaxation properties. The outer zone (zone "M") extends from the core-crust interface, $n_{\mathrm{b}} \sim 0.5 n_{0}$, to some baryon density $n_{\mathrm{D}}$. The relaxation in this zone is provided by the modified Urca processes (b). The intermediate zone "D" extends from the density $n_{\mathrm{D}}$, at which the direct Urca process becomes open, to some density $n_{\mathrm{H}}$. The relaxation goes much faster there, because it is provided by the direct Urca processes (c) while the modified Urca processes are always insignificant once the direct Urca processes are open. The inner zone " $\mathrm{H}$ " extends from the density $n_{\mathrm{H}}$, at which hyperonic processes (d) start to operate, to the stellar center. In this zone, we have the relaxation channels of two very different types: direct Urca (c) and nonlepton hyperonic processes (d), with $\tau_{\mathrm{H}} \ll \tau_{\mathrm{D}}$. The low-mass neutron stars contain only zone M. More massive stars may contain zones $\mathrm{D}$ and $\mathrm{H}$.

In a pulsating star, the baryon number density $n_{\mathrm{b}}$ varies with pulsation period $2 \pi / \omega \sim 10^{-3} \mathrm{~s}$, where $\omega \sim 10^{4} \mathrm{~s}^{-1}$ is a typical pulsation frequency. Even if the unperturbed (non-pulsating) stellar state was in the full equilibrium (which we assume), the pulsating matter may be out of the equilibrium with respect to channels (b)-(d). The adiabatic index for perturbations of the matter has, therefore, to be defined as

$\Gamma_{1}=\frac{n_{\mathrm{b}}}{P}\left(\frac{\mathrm{d} P}{\mathrm{~d} n_{\mathrm{b}}}\right)_{\mathrm{C}}=\frac{\rho+P / c^{2}}{P}\left(\frac{\mathrm{d} P}{\mathrm{~d} \rho}\right)_{\mathrm{C}}$,

where $\rho$ is the mass density and the subscript $\mathrm{C}$ means that the derivative should be constrained by the conditions in various channels (see below). In the full equilibrium, we have $\Gamma_{1}=\Gamma_{\mathrm{EQ}}$, where $\Gamma_{\mathrm{EQ}}$ is the adiabatic index that determines the stiffness of the equation of state (EOS) in a non-pulsating star (i.e., determines the basic neutron-star structure in equilibrium). Figure 1 shows the density dependence of $\Gamma_{\mathrm{EQ}}$ for a relativistic mean-field EOS of Glendenning (1985; his case 3) of npe $\mu \Lambda \Sigma^{-}$matter (similar curve of $\Gamma_{\mathrm{EQ}}$ was calculated, for instance, by Balberg \& Gal 1997). For this EOS, $\mu, \Lambda$, and $\Sigma^{-}$ particles appear at $n_{\mathrm{b}}=0.110,0.310$, and $0.319 \mathrm{fm}^{-3}$, respectively, and the main direct Urca process, Eq. (4), with electrons is allowed at $n_{\mathrm{D}}=0.227 \mathrm{fm}^{-3}$. The adiabatic index $\Gamma_{1}$ in 


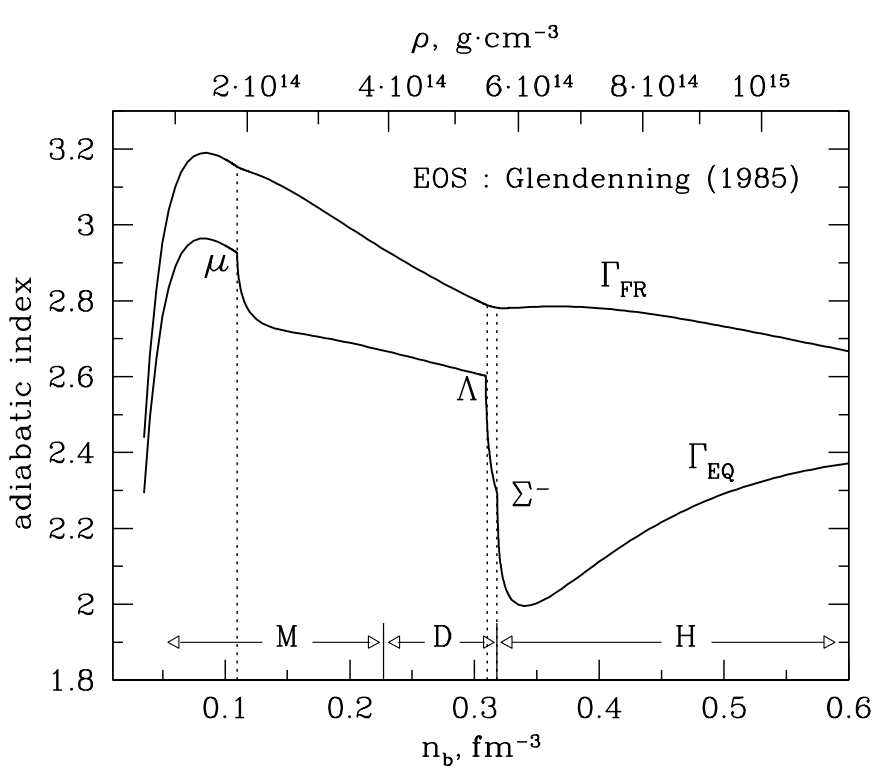

Fig. 1. Adiabatic index $\Gamma_{1}$ versus baryon number density (lower horizontal scale) or mass density (upper horizontal scale) in zones $\mathrm{M}, \mathrm{D}$, and $\mathrm{H}$ (indicated by arrows). The curves show $\Gamma_{\mathrm{EQ}}$ at full equilibrium and $\Gamma_{\mathrm{FR}}$ at fully frozen composition of matter. Vertical dotted lines: the thresholds of appearance of $\mu, \Lambda$, and $\Sigma^{-}$.

question may differ from $\Gamma_{\mathrm{EQ}}$ because of the lack of equilibrium in channels (b)-(d) in pulsating matter.

Exact calculation of $\Gamma_{1}$ is complicated. Thus we restrict ourselves to several important limiting cases.

Generally, the pressure depends not only on $n_{\mathrm{b}}$ but also on the fractions $x_{j}=n_{i} / n_{\mathrm{b}}$ of various particle species $j$. Thus, Eq. (9) can be written as

$\Gamma_{1}=\Gamma_{\mathrm{FR}}+\frac{n_{\mathrm{b}}}{P} \sum_{j}\left(\frac{\partial P}{\partial x_{j}}\right)_{n_{\mathrm{b}}}\left(\frac{\partial x_{j}}{\partial n_{\mathrm{b}}}\right)_{\mathrm{C}}$,

where $\Gamma_{\mathrm{FR}}=\left(\partial \ln P / \partial \ln n_{\mathrm{b}}\right)_{\left\{x_{j}\right\}}$ is the adiabatic index calculated for the "frozen" composition of matter (at $x_{j}=$ constant). The frozen-composition index $\Gamma_{\mathrm{FR}}$ is also depicted in Fig. 1 for the same model of dense matter. Freezing the particle fractions makes an EOS stiffer and increases $\Gamma_{1}$. The real value of $\Gamma_{1}$ lies evidently between $\Gamma_{\mathrm{EQ}}$ and $\Gamma_{\mathrm{FR}}$. Some examples are shown in Fig. 2.

Two obvious conditions of varying the parameters of matter while calculating the derivatives in Eq. (10) are: conservation of baryon number and electric neutrality. This reduces the number of independent fractions (degrees of freedom) by two. Other conditions are described below.

\subsection{Subthermal pulsations in nonsuperfluid matter}

Let us consider nonsuperfluid matter and the subthermal pulsation regime, $\delta \mu_{j} \lesssim T$, for all $j$. In zones $\mathrm{M}$ and $\mathrm{D}$, the relaxation times $\tau_{\mathrm{M}}$ and $\tau_{\mathrm{D}}$ are much larger than the pulsation periods $2 \pi / \omega$. Accordingly, $\Gamma_{1}=\Gamma_{\mathrm{FR}}$ is a good approximation, i.e., $\Gamma_{1}$ is noticeably higher than $\Gamma_{\mathrm{EQ}}$ (Figs. 1 and 2).

In zone $\mathrm{H}$, the situation is more complicated. If the star is sufficiently cold, $T \ll 10^{9} \mathrm{~K}$, we have $\tau_{\mathrm{H}} \gg 2 \pi / \omega$, and again $\Gamma_{1}=\Gamma_{\mathrm{FR}}$. However, in a hotter star, we may have the opposite

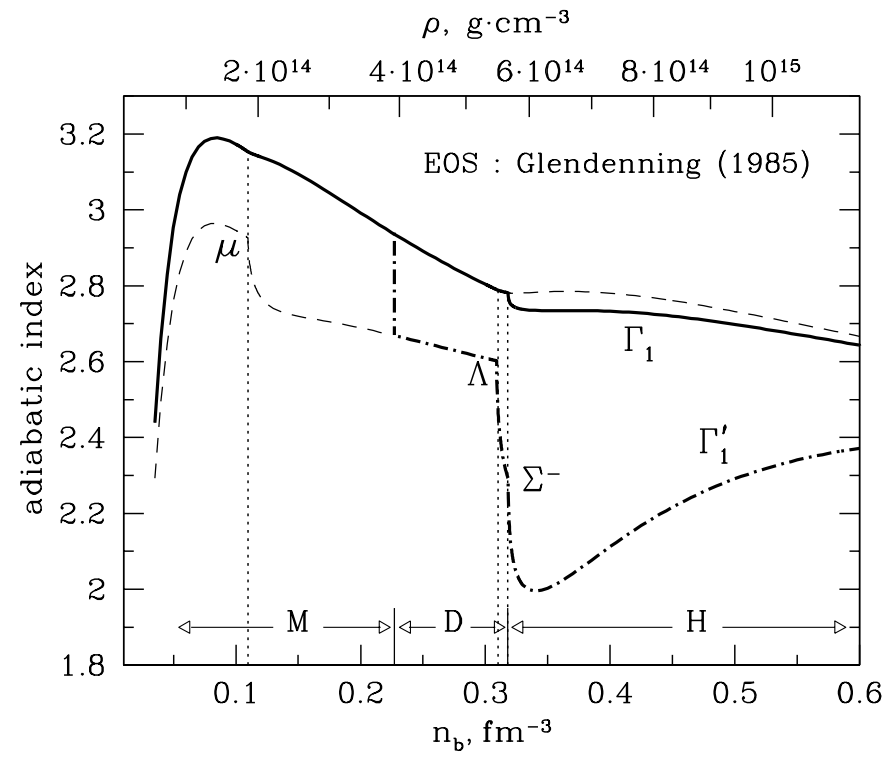

Fig. 2. Same as in Fig. 1 but for different equilibration regimes. Thick solid line: $\Gamma_{1}$ for subthermal perturbations with Urca channels (b) and (c) frozen, but hyperonic channels (d) fully relaxed. Thick dashand-dotted line in zones D and $\mathrm{H}: \Gamma_{1}=\Gamma_{1}^{\prime}$ for suprathermal perturbations with all the channels, but modified Urca, fully relaxed. Thin dashed curves: $\Gamma_{\mathrm{EQ}}$ and $\Gamma_{\mathrm{FR}}$ as in Fig. 1.

condition, $\tau_{\mathrm{H}} \ll 2 \pi / \omega$, which will lead to the equilibrium in hyperonic channels (d); then $\Gamma_{1}$ has to be calculated assuming this equilibrium. The latter implies two conditions:

$\mu_{\mathrm{n}}+\mu_{\Lambda}=\mu_{\mathrm{p}}+\mu_{\Sigma}, \quad x_{\Sigma}+x_{\Lambda}=x_{\Sigma}^{(0)}+x_{\Lambda}^{(0)}$,

in pulsating matter (the upperscript (0) refers to equilibrium values). In combination with conservation of baryon number and electric neutrality in pulsating matter, conditions (11) allow us to calculate the partial derivatives in Eq. (10) and determine $\Gamma_{1}$ (plotted by the thick solid line in Fig. 2). This adiabatic index is only slightly lower than $\Gamma_{\mathrm{FR}}$, for the adopted EOS (cf. the thick solid line and the upper dashed line in Fig. 2).

\subsection{Suprathermal pulsations in nonsuperfluid matter}

Let us outline the case of larger pulsation amplitudes, $\delta \mu_{j} \gg T$, which however are assumed to be relatively small, $\delta \mu_{j} \ll \mu_{j}$, so that the linear pulsation theory is valid. Under such conditions, the deviations of the chemical potentials from the fully equilibrium values will strongly reduce the relaxation times $\tau$. As follows from calculations of the rates of nonequilibrium processes (e.g., Reisenegger 1995; Yakovlev et al. 2001), one can estimate the relaxation time at $\delta \mu_{j} \gg T$ by replacing

$T \rightarrow \widetilde{T}=\sqrt{T^{2}+(\delta \mu)^{2} /(2 \pi)^{2}}$

in Eq. (8), where $\delta \mu$ is a typical pulsation amplitude of chemical potentials. This replacement correctly reproduces the regime of subthermal pulsations ( $\delta \mu \lesssim T, \widetilde{T} \approx T$ ). In the suprathermal regime, $\delta \mu \gg T$, the relaxation times no longer depend on $T$ but depend on the pulsation amplitude $\delta \mu$ instead. Let us stress that the replacement is approximate; detailed calculations are required to obtain quantitatively accurate results in the 
suprathermal regime. Such calculations are beyond the scope of the present paper.

To illustrate the suprathermal effects let us take, for instance, $T=100 \mathrm{keV} \sim 10^{9} \mathrm{~K}, \mu \sim 500 \mathrm{MeV}$, and $\delta \mu \sim 0.02 \mu \sim 10 \mathrm{MeV}$. Then we have $\delta \mu / T \sim 100$ and the relaxation times $\tau_{\mathrm{M}} \sim 0.1 \mathrm{~s}, \tau_{\mathrm{D}} \sim 10^{-4} \mathrm{~s}$, and $\tau_{\mathrm{H}} \sim 3 \times 10^{-6} \mathrm{~s}$. Thus, $\tau_{\mathrm{M}}$ remains much larger than $2 \pi / \omega$, and we have $\Gamma_{1}=$ $\Gamma_{\mathrm{FR}}$, as before, in zone $\mathrm{M}$. However, $\tau_{\mathrm{D}, \mathrm{H}} \ll 2 \pi / \omega$, and we have rapid relaxation in all channels in zones $\mathrm{D}$ and $\mathrm{H}$, where we can put $\Gamma_{1}=\Gamma_{\mathrm{EQ}}$. The adiabatic index for this regime is plotted by the dot-and-dashed line in Fig. 2 (marked as $\Gamma_{1}^{\prime}$ ).

\subsection{Subthermal pulsations in superfluid matter}

Now we return to subthermal pulsations, $\delta \mu \lesssim T$, but assume superfluidity of baryons. All baryon species (n, p, $\Lambda$, and $\Sigma^{-}$, in our example) can be in a superfluid state. The critical temperatures $T_{\mathrm{c}}$ of baryon superfluids depend on the model of strong interactions and the many-body theory employed. Various microscopic models predict density dependent $T_{\mathrm{c}}$, different for different baryons, ranging from $\sim 10^{8} \mathrm{~K}$ to $\sim 10^{10} \mathrm{~K}$ (e.g., Lombardo \& Schulze 2001; also see Haensel et al. 2001, 2002 for references). A strong superfluidity $\left(T_{\mathrm{c}} \gg T\right)$ will drastically (exponentially) reduce the rates of the processes involving baryons (due to the appearance of superfluid gaps in baryon energy spectra; see, e.g., Yakovlev et al. 2001). This will increase the relaxation times (8) in various channels (b)-(d). In this case we can easily have $\tau \gg 2 \pi / \omega$, and the relaxation will be frozen over pulsation periods. Thus, putting $\Gamma_{1}=\Gamma_{\mathrm{FR}}$ will be a good approximation.

\subsection{Suprathermal pulsations in superfluid matter}

This case is complicated. Note however that the pulsations with amplitudes $\delta \mu$ higher than the superfluid gaps, $\Delta \lesssim 1 \mathrm{MeV}$, seem to be independent of superfluid properties of dense matter. Thus, the relaxation times of pulsations with such amplitudes can be estimated using the results of Sect. 3.2.

\section{Damping of stellar pulsations by bulk viscosity}

Let us turn to the bulk viscosity of dense matter and associated damping of neutron-star pulsations. For the composition of dense matter we are interested in, these problems have been studied in a number of papers starting from the pioneering papers by Langer \& Cameron (1969) and Jones (1971). The current state of the problem and references to other papers can be found in Jones (2001), Haensel et al. (2002), and Lindblom \& Owen (2002).

\subsection{Nonsuperfluid matter}

Simple estimates of the bulk viscosity $\zeta$ in the familiar subthermal regime are given, for instance, by Haensel et al. (2001, 2002). The generalization to the case of suprathermal pulsations is straightforward. Generally, one can introduce an effective bulk viscosity $\widetilde{\zeta}$ which determines actual dissipation rate of pulsation energy per unit volume. The estimates of $\widetilde{\zeta}$ can

be obtained from the estimates of $\zeta$ by replacing there $T \rightarrow \widetilde{T}$. In this way we come to the order-of-magnitude estimates of the effective bulk viscosity in zones $\mathrm{M}, \mathrm{D}$, and $\mathrm{H}$ :

$\widetilde{\zeta}_{\mathrm{M}} \sim 5 \times 10^{18} \omega_{4}^{-2} \widetilde{T}_{9}^{6} \mathrm{~g} \mathrm{~cm}^{-1} \mathrm{~s}^{-1}$

$\widetilde{\zeta}_{\mathrm{D}} \sim 5 \times 10^{24} \omega_{4}^{-2} \widetilde{T}_{9}^{4} \mathrm{~g} \mathrm{~cm}^{-1} \mathrm{~s}^{-1}$,

$\widetilde{\zeta}_{\mathrm{H}} \sim 10^{29} \omega_{4}^{-2} \widetilde{T}_{9}^{2} \mathrm{~g} \mathrm{~cm}^{-1} \mathrm{~s}^{-1}$

where $\omega_{4}$ is the pulsation frequency in units of $10^{4} \mathrm{~s}^{-1}$.

At the next step, following Haensel et al. (2002), we estimate a typical viscous dissipation time of stellar pulsations, $t_{\text {diss }} \sim \rho R^{2} / \widetilde{\zeta}$, with $R \sim 10 \mathrm{~km}$ being the radius of the neutronstar core. The timescale $t_{\text {diss }}$ is determined either by dissipation in zone $\mathrm{M}\left(t_{\mathrm{diss}}=t_{\mathrm{M}}\right.$, if zones $\mathrm{D}$ and $\mathrm{H}$ are absent $)$, or dissipation in zone $\mathrm{D}\left(t_{\text {diss }}=t_{\mathrm{D}}\right.$, if zone $\mathrm{H}$ is absent $)$, or dissipation in zone $\mathrm{H}\left(t_{\mathrm{diss}}=t_{\mathrm{H}}\right.$, if a representative zone $\mathrm{H}$ is available $)$. Then we obtain drastically different dissipation times,

$t_{\mathrm{M}} \sim \frac{10 \omega_{4}^{2}}{\widetilde{T}_{9}^{6}} \mathrm{yr}, \quad t_{\mathrm{D}} \sim \frac{100 \omega_{4}^{2}}{\widetilde{T}_{9}^{4}} \mathrm{~s}, \quad t_{\mathrm{H}} \sim \frac{10 \omega_{4}^{2}}{\widetilde{T}_{9}^{2}} \mathrm{~ms}$,

for the different cases.

For the subthermal pulsations, we have $\widetilde{T}=T$ and reproduce thus the estimates given by Haensel et al. (2002). For the suprathermal pulsations, $\delta \mu \gg T$, the effective bulk viscosity and dissipation time (just as the relaxation times in Sect. 3.2) stop to depend on temperature $T$ but depend on $\delta \mu$ instead, producing strongly enhanced nonlinear (in the pulsation amplitude, $\delta \mu$ ) viscous pulsation damping. The damping will completely destroy the pulsation mode if the dissipation time $t_{\text {diss }}$ becomes comparable to the pulsation period $2 \pi / \omega$. Thus, setting $t_{\text {diss }}=2 \pi / \omega$ we can find the maximum pulsation amplitude $\delta \mu_{\max }$ which a pulsating star can sustain. For the pulsations in regimes $\mathrm{M}, \mathrm{D}$, and $\mathrm{H}$, we obtain:

$\delta \mu_{\max }^{\mathrm{M}} \sim 50 \omega_{4}^{1 / 2} \mathrm{MeV}$

$\delta \mu_{\max }^{\mathrm{D}} \sim 10 \omega_{4}^{3 / 4} \mathrm{MeV}$,

$\delta \mu_{\max }^{\mathrm{H}} \sim 2 \omega_{4}^{3 / 2} \mathrm{MeV}$.

Accordingly, the viscous damping cannot allow the star to pulsate with too large amplitude, and the maximum amplitude does depend on the damping regime. It is natural to assume that the strong damping with the maximum amplitude will rapidly reduce the pulsation amplitudes to a lower level with a smaller damping rate.

\subsection{Superfluid matter}

The effects of superfluidity are similar to those described in Sects. 3.3 and 3.4. For subthermal pulsations, these effects were considered, for instance, by Haensel et al. (2000, 2001, 2002). A strong superfluidity may drastically (by many orders of magnitude) reduce the bulk viscosity and the dissipation rate. The effects of superfluidity on the bulk viscosity and the damping of pulsations in the suprathermal regime with $\delta \mu \gtrsim \Delta$ are expected to be small, i.e., the results of Sect. 4.1 can be used. 


\section{Summary}

We have outlined the most important factors which affect the adiabatic index $\Gamma_{1}$ for density perturbations and the viscous damping of pulsations in the neutron-star cores. First, nonleptonic strangeness-changing processes with hyperons may be rapid enough to establish partial hyperonic equilibrium over pulsation periods and decrease $\Gamma_{1}$ slightly below the "frozen" adiabatic index, $\Gamma_{\mathrm{FR}}$, in hyperonic matter. In addition, they induce a rapid viscous damping even in the subthermal pulsation regime $\left(\delta \mu_{j} \lesssim T\right)$. Second, suprathermal but still linear perturbations $\left(T \ll \delta \mu_{j} \ll \mu_{j}\right.$ ) may produce a very rapid equilibration in various relaxation channels which reduces $\Gamma_{1}$ to the fully equilibrium value $\Gamma_{\mathrm{EQ}}$, enhances the bulk viscosity and damps the pulsations quickly to lower amplitudes. Third, superfluidity of baryons in the subthermal regime increases relaxation times in various channels, brings $\Gamma_{1}$ closer to $\Gamma_{\mathrm{FR}}$, and reduces the viscous dissipation.

Therefore, a proper calculation of neutron star pulsations and their dynamical evolution represents a complicated problem. Much work is required to study this problem in full detail. Our order-of-magnitude estimates have to be replaced with accurate numerical solutions of the equations of stellar pulsations taking into account proper boundary conditions and joint effect of various factors in all neutron-star layers, from the surface to the center. Our assumption of one typical pulsation amplitude of chemical potentials, $\delta \mu$, in Eq. (12) is an oversimplification. In reality, one has to deal with the number of density dependent amplitudes $\delta \mu_{j}$ for different particle species $j$. The relaxation in different equilibration channels and the viscous damping of pulsations can be strongly nonuniform; one cannot exclude the existence of thin layers in the neutron star cores, where the damping is exceptionally strong. For instance, they may be the layers where new hyperons appear, with sufficiently small chemical potentials $\mu_{j}$ just beyond their appearance threshold. Neutron star pulsations in these layers may be suprathermal, producing enhanced damping. The problem of suprathermal pulsation regime, without and with superfluidity of baryons, is of special importance. This regime will be accompanied by huge energy release which will heat the star.
Acknowledgements. DGY is grateful to A. A. Pamyatnykh for a helpful discussion. KPL and DGY acknowledge hospitality of $\mathrm{N}$. Copernicus Astronomical Center in Warsaw. This work was supported in part by the RBRF (grants Nos. 02-02-17668 and 00-07-90183) and KBN (grant 5 P03D 020 20).

\section{References}

Balberg, S., \& Gal, A. 1997, Nucl. Phys. A, 625, 435

Chanmugan, G., \& Gabriel, M. 1971, A\&A, 13, 374

Chanmugan, G. 1977, ApJ, 217, 799

Flowers, E., \& Itoh, N. 1979, ApJ, 230, 847

Glendenning, N. K. 1985, ApJ, 293, 470

Gourgoulhon, E., Haensel, P., \& Gondek, D. 1995, A\&A, 294, 747

Gondek, D., Haensel, P., \& Zdunik, J. L. 1997, A\&A, 325, 217

Haensel, P., Levenfish, K. P., \& Yakovlev, D. G. 2000, A\&A, 357, 1157

Haensel, P., Levenfish, K. P., \& Yakovlev, D. G. 2001, A\&A, 372, 130

Haensel, P., Levenfish, K. P., \& Yakovlev, D. G. 2002, A\&A, 381, 1080

Jones, P. B. 1971, Proc. Roy. Soc. Lond., 323, 111

Jones, P. B. 2001, Phys. Rev. D64, 084003

Langer, W. D., \& Cameron, A. G. W. 1969, Astrophys. Space Sci., 5, 213

Lindblom, L., \& Owen, B. J. 2002, Phys. Rev. D, 65, 063006

Lombardo, U., \& Schulze, H.-J. 2001, in Physics of Neutron Star Interiors, ed. D. Blaschke, N. Glendenning, \& A. Sedrakian (Springer, Berlin), 31

Meltzner, D. W., \& Thorne, K. S. 1966, ApJ, 145, 514

Shapiro, S. L., \& Teukolsky, S. A. 1983, Black Holes, White Dwarfs and Neutron Stars (Wiley-Interscience, New-York)

Reisenegger, A. 1995, ApJ, 442, 749

Thorne, K. S., \& Campolattaro, A. 1967, ApJ, 149, 591 (erratum: $1968,152,673)$

Thorne, K. S. 1968, Phys. Rev. Lett., 21, 320

Thorne, K. S. 1969a, ApJ, 158, 1

Thorne, K. S. 1969b, ApJ, 158, 997

Yakovlev, D. G., Kaminker, A. D., Gnedin, O. Y., \& Haensel, P. 2001, Phys. Rep., 354, 1 